\title{
Efecto antifúngico de cuatro mezclas de monoterpenos como alternativa para el control de Rhizoctonia solani Kün.
}

\author{
(Antifungal effect of four mixtures from monoterpenes like alternative by control \\ of Rhizoctonia solani Kün).
}

Daymara I Vaillant F1; Carlos R Romeu C'; Rebeca Ramírez $0^{1}$.

Instituto de investigación de Sanidad Vegetal 110 \# 514 e/ 5ta B y 5ta F, Playa, Ciudad de La Habana. Autor para correspondencia: dvaillant@inisav.cu

RECIBIDO:7 de Febrero de 2015

APROBAD0:10 de Mayo de 2015

LOS AUTORES DECLARAN NO TENER CONFLICTO DE INTERESES

Palabras claves: actividad fungicida, monoterpenos, Rhizoctonia solani.

Key words: fungicide activity, monoterpenes, Rhizoctonia solani.

\section{RESUMEN}

Rhizoctonia solani Kühn, es un patógeno de suelo, que causa enfermedades en un amplio rango de hospedantes de cultivos agrícolas. Las necesidades de reducir el uso indiscriminado de fungicidas químicos conllevan al estudio de otras alternativas menos agresivas al medio ambiente. Los monoterpenos presentes en los aceites esenciales de plantas, presentan una marcada actividad biocida y son compuestos que resultan menos perjudiciales desde el punto de vista ambiental. Se prepararon cuatro mezclas de monoterpenos (timol-mentol, timolalcanfor, timol-citronelal y timol-1,8 cineol) con el objetivo de evaluar la actividad antifúngica in vitro sobre $R$. solani, mediante su efecto sinérgico. Se empleó el método de adición al medio de cultivo de cada una de las mezclas a concentraciones de: 0,5; 0,$1 ; 0,05 ; 0,03$ y $0,01 \%$, partiendo de una solución madre al 10\% en dimetilsufóxido (DMSO) al $5 \%$. Se calcularon los porcentajes de inhibición y los resultados se analizaron estadísticamente. Se determinaron las dosis inhibitorias medias y se clasificaron la toxicidad de cada una de las mezclas en: inocua, ligera, moderada y tóxica a cada concentración. Las cuatro mezclas mostraron 100\% de inhibición a las concentraciones de: 0,$5 ; 0,1$; 0,05 y $0,03 \%$. A la menor concentración las mezclas de timol-mentol y timol-cineol fueron las de mayor efecto inhibitorio del crecimiento micelial de $R$. solani.

\section{ABSTRACT}

Rhizoctonia solani Kühn is a soil pathogen that causes diseases in a wide host range from agricultural crops. The need to reduce the indiscriminate use of chemical fungicide it has led to the study of other less aggressive environment alternatives. Monoterpenes, common components in plants essential oils, shows a maked biocide activity and are compounds less harmful from an environmental point of view. With the objective of evaluate the in vitro antifungal activity against $R$. solani, four binary monoterpenes mixtures (thymol-menthol, thymol-camphor, thymol-citronellal and thymol-1,8 cineol) with the objective of evaluate the in vitro antifungal activity against $R$. solani, four monoterpenes mixtures were prepared. The method of addition to culture media of each of the mixtures in concentrations of: 0,$5 ; 0,1 ; 0,05 ; 0,03$ and $0,01 \%$, from a stock solution to $10 \%$ in dimethylsulfoxide (DMSO) to $5 \%$ were used. The inhibition percentages were calculated and the results were statistically 
analyzed. Median inhibitory dose was determined and the toxicity of each mixture were classified in: harmless, light, moderate and toxic in each concentration. The four mixtures showed $100 \%$ of micelial growth inhibition in concentrations of 0,$5 ; 0,1 ; 0,05$ and $0,03 \%$. The thymol-cineol and thymol-menthol mixtures had the higher inhibitory effect on micelial growth of $R$. solani at the lower concentration.

\section{INTRODUCCIÓN}

Rhizoctonia solani Kühn es un patógeno de distribución cosmopolita nativo del suelo, que afecta a un gran número de hospederos, sobre todo en cultivos anuales (Meza et al., 2007; Murillo, 2013). Para el control de este hongo en muchos países, incluyendo Cuba, se aplican diversos fungicidas de acción protectora (como los del grupo de las carboxamidas) (Almandoz et al., 2000; Murillo, 2013). Debido al impacto negativo que provocan los fungicidas químicos tanto en la biodiversidad de los agroecosistemas y en la salud pública; se encamina a la búsqueda y desarrollo de sustancias naturales con actividad antibiótica, como alternativas ecológicas (Hernández et al., 2010). Una de los más actuales es el uso de productos derivados de las plantas; entre los que se encuentran aceites esenciales, terpenos, lignanos, alcaloides, azúcares, esteroides, entre otros (Guijarro y García, 2014). Dixon (2001) informa que estas sustancias tienen un rol importante en el mecanismo defensivo de las plantas. Por lo tanto, en Ios últimos años se está retornando al uso de las plantas como fuente de plaguicidas más seguros para el medio ambiente y la salud humana (Ottaway, 2001; Beltrán et al., 2013).

Los aceites esenciales son productos del metabolismo secundario de las plantas y constituyen el resultado del balance entre su biosíntesis, su catabolismo y/o pérdida física (Hadi, 2014). Estos productos son mezclas muy complejas de alrededor de 50 compuestos como; monoterpenos, sesquiterpenos, aldehídos, cetonas y fenoles (Isman et al., 2011). Los monoterpenos son las moléculas más abundantes, llegando a representar hasta el 90\% del aceite (Hernández, 2011). Estos compuestos que resultan menos perjudiciales desde el punto de vista ambiental; se conoce que presenta una notable actividad fungicida, fundamentalmente cuando se combinan entre ellos. (Saccheti et al., 2005).

Se poseen cuatro mezclas de monoterpenos, los cuales se probaron con anterioridad de forma independiente cada uno, mostrando actividad fungicida (Vaillant et al., 2009). A partir del resultado obtenido se propone: evaluar el efecto antifúngico in vitro de cada una de las mezclas, mediante su acción sinérgica sobre una cepa de $R$. solani utilizando diferentes concentraciones de los productos.

\section{MATERIALES Y MÉTODOS}

Se emplearon cuatro mezclas binarias de monoterpenos presentes en aceites esenciales (timolmentol, timol-alcanfor, timol-citronelal y timol-1,8 cineol). Para el análisis de la antibiosis se utilizó una cepa de Rhizoctonia solani (Rorn2) obtenida de papa, debidamente conservado a $4 \mathrm{C}^{\circ}$ en cuñas de PDA (10 réplicas) perteneciente a la colección del cepario del laboratorio de micología del Instituto de Investigaciones de Sanidad Vegetal (INISAV).

Se prepararon cinco soluciones de las mezclas a las concentraciones de 0,$5 ; 0,1 ; 0,05 ; 0,03$ y $0,01 \%$ en medio de cultivo agar papa dextrosa (PDA), a partir de una solución madre al 10\% en DMSO al 5\%. Las soluciones se extendieron en placas Petri de $9 \mathrm{~cm}$ de diámetro, se emplearon dos controles negativos, uno de crecimiento en PDA sin tratamiento y otro con DMSO al 0,5\%. Se sembraron en el centro de las placas discos de $5 \mathrm{~mm}$ de diámetro del hongo y se incubaron a $28 \mathrm{C}^{\circ}$ durante cinco días. Se emplearon tres réplicas por tratamiento. Pasado el tiempo de incubación se midió el diámetro de las colonias tratadas y la colonia control, y se calculó el porciento de inhibición por la siguiente fórmula:

\section{$\%$ inhibición $=[(D C C-D C T) / D C C] \times 100$}

(DCC: diámetro de la colonia control DCT: diámetro de la colonia tratada).

Los datos anteriores se procesaron mediante análisis de varianza de clasificación simple. (ANOVA) utilizando el programa Analest (versión 2.0). Las medias se docimaron mediante la Prueba de Tuckey $(p<0.05)$. 
Se determinaron los niveles de toxicidad de las mezclas de monoterpenos según los resultados de los porcentajes de inhibición. Para esto se siguió la escala establecida por la Organización Internacional de Lucha Biológica (OILB) (Tabla 1).

Tabla 1. Clasificación establecida a escala de laboratorio a productos con actividad antimicrobiana (Viñuela y Jacas, 1993).

\begin{tabular}{|l|l|}
\hline $\begin{array}{l}\text { Porcentajes de inhibición } \\
\text { del crecimiento micelial }\end{array}$ & Clasificación \\
\hline Valores $<30 \%$ de mortalidad & Inocuo \\
\hline Valores $30-79 \%$ de mortalidad & Ligeramente tóxico \\
\hline Valores $80-99 \%$ de mortalidad & Moderadamente tóxico \\
\hline Valores $>99 \%$. & Tóxico \\
\hline
\end{tabular}

\section{RESULTADOS}

Las cuatro mezclas de monoterpenos mostraron un $100 \%$ de inhibición a las concentraciones de: 0,5; 0 , $1 ; 0,05$ y $0,03 \%$, manifestando así un efecto fungicida sobre $R$. solani (figura 1.)

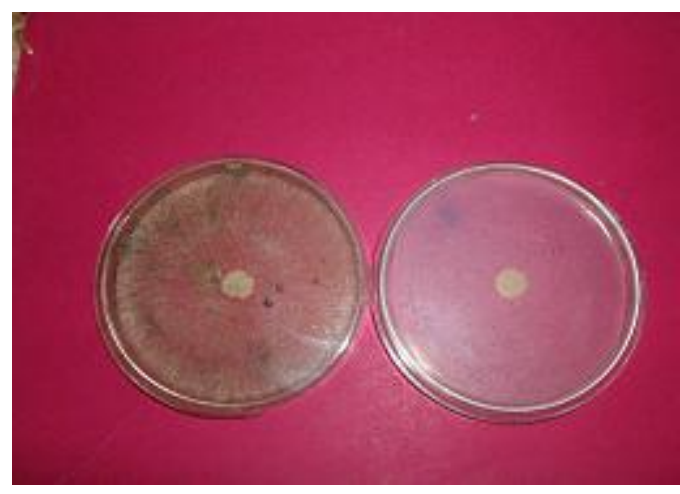

Fig. 1. Efecto inhibitorio de la mezcla timol-citronelal sobre Rom2. A la izquierda se muestra $R$. solani creciendo sin el producto y a la derecha el hongo con citronel al al 0,1\%.
Al $0,01 \%$ las mezclas de timol-mentol y timolcineol obtuvieron un valor de un $70 \%$ de inhibición. La mezcla timol-citronelal inhibió el crecimiento del hongo en un $52 \%$, mientras que el timol-alcanfor fue el de menor porcentaje con valores por debajo de un $40 \%$, todos con diferencias significativas con el control negativo (Figura2). Estos resultados denotan que al realizarse combinaciones binarias de monoterpenos, el efecto antifúngico de cada uno aumenta. Vaillant et al. (2009) realizaron estudios de la actividad fungicida de estos monoterpenos. El 1,8 cineol y el alcanfor al 0,05\% fueron los de más bajo porcentaje de inhibición frente a la cepa Rorn2; los valores de estos monoterpenos fueron tan bajos que en el caso del alcanfor no mostró diferencias con el control negativo, sin embargo en este estudio al combinarse con el timol, su efecto fungicida aumentó en $100 \%$ de inhibición. Resultados similares se obtuvieron con el mentol.

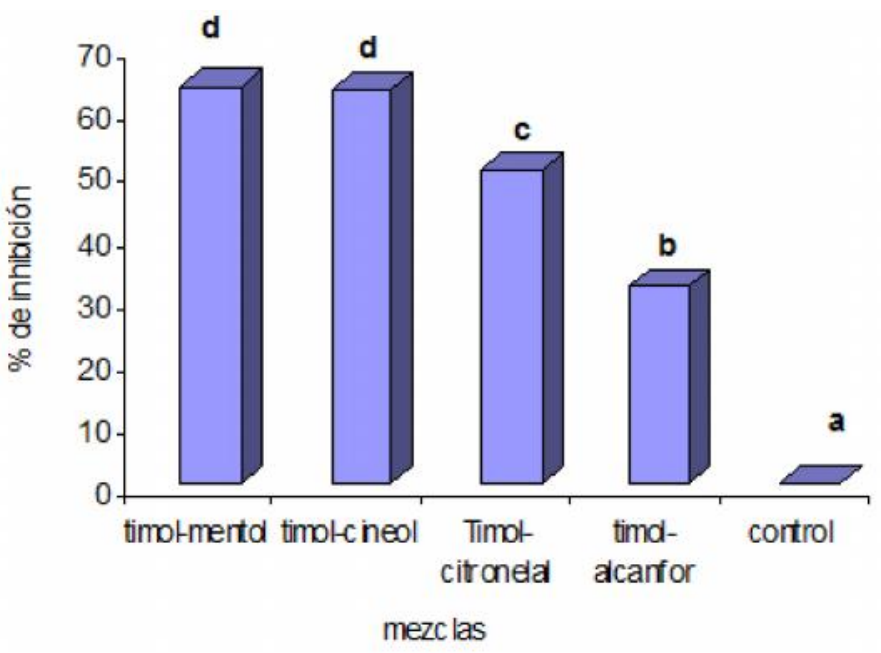

Fig. 2. Porcientaje de inhibición de cada mezcla al $0,01 \%$

Las cuatro mezclas estudiadas fueron tóxicas para $R$. solani a las concentraciones de: 0,5; 0, 1; 0,05 y $0,03 \%$. Al 0,01\% todas las mezclas fueron ligeramente tóxicas (Tabla 2). 
Tabla 2. Clasificación de la toxicidad de las mezclas de monoterpeno frente a Rorn2

\begin{tabular}{|l|c|l|l|l|l|}
\hline Mezclas & $\mathbf{0 , 5} \%$ & $\mathbf{0 , 1} \%$ & $\mathbf{0 , 0 5 \%}$ & $\mathbf{0 , 0 3 \%}$ & $\mathbf{0 , 0 1 \%}$ \\
\hline Timol-mentol & Tóxico & Tóxico & Tóxico & Tóxico & Ligeramente tóxico \\
Timol-cineol & Tóxico & Tóxico & Tóxico & Tóxico & Ligeramente tóxico \\
Timol-citronelal & Tóxico & Tóxico & Tóxico & Tóxico & Ligeramente tóxico \\
Timol-alcanfor & Tóxico & Tóxico & Tóxico & Tóxico & Ligeramente tóxico \\
\hline
\end{tabular}

\section{DISCUSIÓN}

Son varios los autores que hacen referencia a la actividad biocida de los aceites esenciales, debido al efecto sinérgico de sus componentes. El aceite esencial de Thymus zygis presenta como compuestos activos al timol y carvacrol; este aceite mostró actividad fungicida sobre Pythium irregulare y $R$. solani (Pérez, 2012). Dubey y Kishore (1987) encontraron que la combinación de tres extractos de aceites esenciales de diferentes plantas tuvieron un efecto inhibidor del crecimiento de 15 hongos, con resultados significativos en: Alternaria solani, Fusarium moniliforme y F. oxysporum (Zapata et al., 2010).

Por otra parte el timol como componente aislado del aceite esencial, tiene efecto fungistático sobre varias especies de hongos fitopatógenos tales como: Phytopthora capsici, P. citrophthora, Penicillium italicum y P. expansum (Camele et al., 2012). Estudios realizados por Velásquez et al., 2014, mostraron que el timol era capaz de inhibir en un $100 \%$ el crecimiento micelial de Penicillium digitatum a la concentración de $50 \mathrm{ppm}$, y frente a $P$. italicum inhibieron su crecimiento en un $82 \%$. También se tienen informes acerca de productos cuyo principio activo es el timol, los cuales manifiestan resultados significativos en el control de enfermedades tanto bacterianas como fúngicas, debido principalmente a la unión con otros componentes minoritarios (Giovinazzo et al., 2012). Otros autores observaron que el efecto inhibidor del aceite esencial de Timbra spicata sobre $R$. solani se debía a la presencia del timol (Sanabria y Rodríguez, 2005).
El 1,8 cineol como componente, no se ha empleado como fungicida según la literatura consultada. Este monoterpeno es conocido por su potencial alelopático contra malezas y su capacidad de repeler insectos, gusanos y agentes vectores de enfermedades (Maggi, 2004). Además, el 1,8 cineol está presente como componente mayoritario en el aceite esencial de muchas especies del género Salvia (Santana et al., 2012). Lo mismo ocurre con el citronelal el cual se utiliza en la preparación de insecticidas a base de aceites esenciales. Este monoterpeno es componente mayoritario, conjunto con el 1-8 cineol, del aceite esencial de especies del género Eucaliptus y se conoce que presenta actividad herbicida frente arvenses (González, 2011). Sin embargo en este estudio ambos monoterpenos manifestaron porcentajes de inhibición micelial significativos, cuando eran combinados con el timol.

Algunos autores plantean que la actividad antimicrobiana combinada de varios monoterpenos está relacionada con su composición química, la proporción en que se encuentren estos componentes en los aceites esenciales y la interacción entre ellos (Dorman y Deans, 2000; Marino et al., 2001; Delaquis et al., 2002 citado por: Hernández, 2011). Lambert et al. (2001) afirman que los dos componentes mayoritarios del aceite esencial de orégano; carvacrol y timol aumentaron su efecto antimicrobiano cuando se usaban combinados frente a Staphylococcus aureus y Pseudomonas aeruginosa (Hernández, 2011).

En los últimos años se realizaron estudios sobre 
los posibles mecanismos de acción de los aceites esenciales y sus componentes monoterpénicos. Entre estos estudios se pueden citar los efectuados por: Rasooli y Owlia (2005) los cuales exponen que los aceites esenciales de Thymus eriocalyx y Thymus $X$ porlock cuyo componente mayoritario es el timol, pueden provocar en Aspergillus parasiticus daño irreversible de la pared celular, la membrana celular y organelos celulares (Barrera y García, 2008). Este efecto no solo es provocado por un solo compuesto del aceite esencial sino por la mezcla de varios productos presentes en dicho aceite (Hashema et al., 2010).

Los resultados obtenidos en este trabajo permiten constatar el efecto sinérgico que pueden presentar estos monoterpenos una vez que se combinan. Esto permitiría continuar estudios de actividad fungicida, a partir de aceites esenciales de plantas que presenten estos compuestos.

\section{CONCLUSIONES}

Las concentraciones más efectivas fueron 0,$5 ; 0,1$; 0,05 y $0,03 \%$ donde hubo $100 \%$ de inhibición y se obtuvo en efecto fungicida sobre $R$. solani.

Las mezclas timol-citronelal y timol-mentol fueron las más eficaces frente $R$. solani, a la concentración de $0,01 \%$.

Exceptuando la concentración de 0,01\% que fue ligeramente tóxica, las restantes concentraciones fueron clasificadas de tóxicas para $R$. solani.

\section{REFERENCIAS}

1. Almandoz, J. E.; Pico, V. M.; Pérez, L.; Rodríguez, F.; Parra, J. (2000). Efectividad de nuevos fungicidas para el control de Alternaria solani Ellisy Martin en el cultivo de la papa (Solanum tuberosum L.). XIX Congreso de la Asociación Latinoamericana de la Papa (ALAP), 28de febrero a 3 de marzo, La Habana. Cuba en: www.inisav.cu (Consultado: septiembre, 2014).

2. Barrera, L L.; García, L J. (2008). Actividad antifúngica de aceites esenciales y sus componentes sobre el crecimiento de Fusarium sp aislado de papaya (Carica papaya). Revista UDO Agrícola: 8 (1): 33-41.

3. Camele, I.; Altieri, L.; De Martino, L.; De Feo, V.; Mancini, E.; Rana, G .L. (2012). In vitro control of post-harvest fruit rot fungi by some plant essential oil components. International Journal of Molecular Science 13 (2): 2290-2300.

4. Dixon, R. (2001). Natural Products and Plant Disease Resistance. Nature 411: 843-847.

5. Giovinazzo, G.; Ingrosso, I.; Paradiso, A.; DeGara, L.; Santino, A. (2012). Resveratrol biosynthesis: plant metabolic engineering for nutritional improvement of food. Original paper. Journal Plant Foods for Human Nutrition 67(3): 191-199.
6. González, L.E. (2011). Efectos del aceite esencial y extractos acuosos de Eucalyptus gomphocephala DC sobre la germinación y el crecimiento de arvenses. Tesis para la opción de Máster en producción de vegetal y ecosistemas agroforestales. Escuela técnica superior de ingeniería agronómica y del medio natural. Universidad politécnica de Valencia. 42 p, España en: https://riunet.upv.es (Consultado septiembre, 2014).

7. Guijarro, G.; García, A. (2014). Alternativas a los antibióticos en la producción de porcino (1). SUIS, 109: 14-20.

8. Hadi, A. (2014). Principios con actividad antifúngica de la raíz y rizoma de Ferula hermonis. Tesis de poción del título de Doctor en Farmacología. Departamento de farmacología, de terapéutica y de toxicología, Unidad Docente Hospital Universitario Germans Trias I Pujol. Universidad Autónoma de Barcelona. Barcelona, España. 176p, 2014 en: www.ddd.uab.cat. (Consultado septiembre, 2014).

9. BHashema, M.; Moharama, A M.; Zaiedb, A A.; Salehb, F.E.M. (2010). Efficacy of essential oils in the control of cumin root rot disease caused by Fusarium spp. Crop Protection. 29: 1111-1117. 
10. Hernández, M.; Hernández, F. D.; Lira, R. H.; Gallegos, G . (2010). Biocontrol de Rhizoctonia solani y Fusarium sp. con Microencapsulados de Bacillus subtilis y su Efecto en Crecimiento y Rendimiento de Tomate (Lycopersicon esculentum Mill. Revista Agraria. 7 (1, 2,3): 17-25, México, 2010.

11. Hernández, P. (2011). Encapsulación de aceite esencial de clavo para su aplicación en la industria alimentaria. Tesis para la opción del título de Doctor en Ciencias. Facultad de ciencias de la salud, de la actividad física y del deporte. Universidad católica de San Antonio. Murcia, España. 276 p, 2011 en: http: / /www.repositorio.ucam.edu (Consultado septiembre, 2014).

12. Isman, M. B.; Miresmailli, S.; Machial, C. (2011). Commercial opportunities for pesticide based on plan essential oils in agriculture, industry and consumer products», Phytochem. Rev. 10: 197-204.

13. Lambert, R J W.; Skandam, P N.; Cute, P J.; Nychas, G J E. (2001). Astudy of the minimum inhibitory concentration carvacol. Journal of Applied Microbilogy. 91 453-462.

14. NMaggi, M. E. (2004). Insecticidas naturales. En: http://www.monografias.com/ (consultado 4 de septiembre del 2008).

15. Meza, M.; González, N.; A. Usubillaga. (2007). Composición del aceite esencial de Origanum majorana L. extraído por diferentes técnicas y su actividad biológica. Rev. Fac. Agron. (LUZ). 24: 725-738.

16. Murillo, W.; Araque, P.; Henao, B.; Peláez, C. (2013). Actividad insecticida de una emulsión aceite/agua del aceite esencial de Eucalyptus tereticornis. Revista Cubana de Plantas Medicinales. 18(1) 109-117.

17. Ottaway, P. B. (2001). The roots of a healthy diet. Chem Ind (22): 42-44.

18. Pérez, R.; J. Ubera. (2006). Fungitoxic activity against phytopathogenic fungi and the chemical composition of Thymus zygis essential oils in South Iberian Peninsula. I Congreso sobre Seguridad Alimentaria. CIBSA, 2006. Sevilla, España. Formato Digital en: www.redsicura.iata.csic.es. última consulta enero, 2012.
19. Rasooli, I.; Owlia, P. (2005). Chemoprevention by thyme oils of Aspergillus parasiticus growth and aflatoxin production. Phytochemistry. 66(24):28512856.

20. Sacchetti, G.; Maietti, S.; Muzzoli, M.; Scaglianti, M.; Manfredini, S.; Radice, M, Bruni, R. (2005). Comparative evaluation of 11 essential oils of different origin as functional and spatial analysis of epidemics caused by species in the genus antioxidants, antiradicals and antimicrobials in foods. Food Chem. 91: 621-632.

21. Sanabria, M.; Rodríguez, D. (2005). Efecto del extracto de tres plantas silvestres sobre la rizoctoniasis, la mancha sureña del maíz y los patógenos que la causan. Interciencia. 30:793-744.

22. Santana, 0.; Cabrerac, R.; Giménezc, C.; González, A.; Sánchez, de los Mozos, R.; Rodríguez, M.; Laserna-Ruiza, I.; Usano, J.; Herraiza, D. (2012). Perfil químico y biológico de aceites esenciales de plantas aromáticas de interés agro-industrial en Castilla-La Mancha (España). Grasas y aceites. 63(2): 214-222.

23. Vaillant, D.; Romeu, C.; Ramos, E.; González, M.; Ramírez, R.; González, J. (2009). Efecto inhibitorio in vitro de cinco monoterpenos de aceites esenciales sobre un aislado de Rhizoctonia solani en papa (Solanum tuberosum L.). Fitosanidad; 13 (3): 197200.

24. Velásquez, M.; Álvarez, R.; Tamayo, P.; Carvalho, C. (2014). Evaluación in vitro de la actividad fungistática del aceite esencial de mandarina sobre el crecimiento de Penicillium sp. Corpoica Cienc. Tecnol. Agropecu. 15(1) 7-14.

25. Viñuela, E.; Jacas, J.(1993). Los enemigos naturales de las plagas y los plaguicidas. Ministerio de Agricultura. H.D. 2-93. Madrid. 24 pp.

26. Zapata, B.; Duran, C.; Stashenko, E.; BetancurGalvis, L.; Mesa, A C. (2010). Actividad antimicótica y citotóxica de aceites esenciales de plantas de la familia Asteraceae. Rev Iberoam Micol. 27 (2):101-103. 